\title{
The Relative Risk Estimation of Pneumonia in Malaysia using Standardized Morbidity Ratio (SMR)
}

\author{
Ijlal Mohd Diah ${ }^{1}$ and Nazrina Azizi, ${ }^{1 *}$ \\ ${ }^{1}$ Department of Mathematics and Statistics, School of Quantitative Sciences, UUM College of Arts and Sciences, \\ Universiti Utara Malaysia, 06010, Sintok, Kedah Darul Aman, Malaysia \\ ${ }^{2}$ Institute of Strategic Industrial Decision Modelling (ISIDM),Universiti Utara Malaysia, 06010, Sintok, Kedah Darul
}

\begin{abstract}
Pneumonia is the most serious inflammatory disease of the respiratory system which cause millions of deaths worldwide especially for children under age five and older adults. The numbers of pneumonia cases reported keep on increasing from year to year in Malaysia. Moreover, this type of disease can become outbreak if it is not controlled. Besides using as prevention strategies for a disease risk, statistical modelling of disease mapping can also be used in monitoring as it clearly shows risk areas. The objective of this paper is to estimate the value of relative risk for pneumonia transmission by using Standardized Morbidity Ratio (SMR) method which is the conventional statistic method used in disease mapping and subsequently propose a disease risk map to show the pneumonia risk areas. Pneumonia data in Malaysia is used in estimating the relative risk. The results show that, Terengganu has the highest risk of contracting pneumonia while Pulau Pinang shows the lowest risk.
\end{abstract}

Keywords: disease mapping; pneumonia; Standardized Morbidity Ratio; relative risk

\section{INTRODUCTION}

Pneumonia is an infection in the lung when alveoli are filled up with inflammatory cells (Otieno et al., 2012). According to Otieno et al. (2012), the disease can be caused by microorganisms such as parasites, fungi, bacteria and viruses. Bacteria has been recognized to be the main cause that lead someone to get pneumonia, especially streptococcus pneumoniae (pneumococcus) (Otieno et al., 2012). General symptoms for those who have contact with pneumonia will have high grade fever, chills, sweating, coughing with yellow or green or blood-stained sputum, shortness of breath and chest pain. The cough may be dry or with sputum depending upon the germs that caused pneumonia at the first place (Rahil, 2019).

Pneumonia can be spread via air-borne when someone who has been infected sneezing or coughing (WHO, 2019). Tiny droplets containing bacteria are released into the air. According to WHO (2019), someone will also get pneumonia from either bacteria or viruses that are already in their nose or throat, which can usually be found in children.

Pneumonia can be treated with antibiotics. Amoxicillin dispersible tablets are the choice of antibiotic recommended (WHO, 2019). In most cases, oral antibiotics can be used in diagnosing and treating the pneumonia. However, for severe cases of pneumonia, hospitalization of patient might be needed (WHO, 2019). As prevention for children under the age of two, pneumococcal vaccines are advisable to be taken for them. This can help to reduce the risk for them to get pneumonia disease.

Pneumonia is ranked among the top three diseases that cause death in adults and children under five in Malaysia (Department of Statistics Malaysia, 2019). Based on Figure 1, pneumonia is the second cause of deaths in year 2017 and 2018 with $12.7 \%$ and $11.8 \%$, respectively. It is also the main cause of deaths for age o until 14 years old in 2017 and 2018 with $4.1 \%$ and $4.8 \%$, respectively (Department of Statistics Malaysia, 2019). In 2019, the total number of cases recorded 
is 145419 cases with 7542 number of death due to this disease.

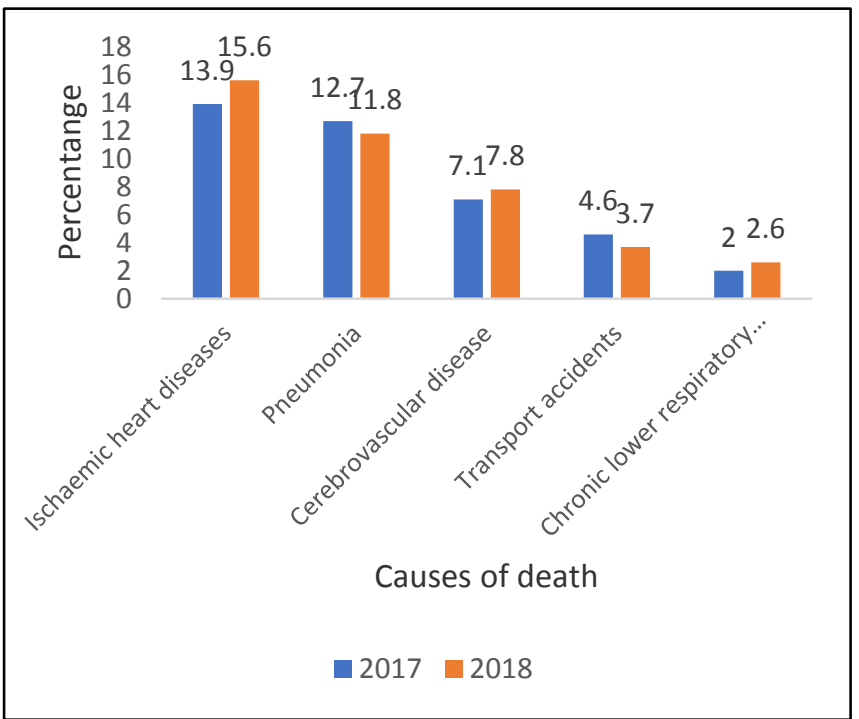

Figure 1. Five principal causes of deaths in Malaysia for the year 2017 and 2018

It is necessary to look into this problem as complication for those who are infected with influenza and Corona Virus Disease 2019 (COVID-19) can lead others to contract pneumonia disease (WHO, 2020). Both can contribute to the increase in the number of pneumonia cases. Pneumonia disease might become an outbreak if it is not controlled and curbed.

Disease mapping is one of the methods that can be used in order to monitor disease or as the prevention strategies by public health and government. Disease mapping can be seen as a descriptive picture of pneumonia's burden in some geographical areas. Besides, it can help to show areas that need more attention from government especially in term of health supply and medical treatment. The disease mapping can clearly demonstrate the risk areas, which is good in monitoring the disease incidence and used as the prevention strategies (Samat \& Percy, 2012). Better estimates of relative risk will subsequently review a true risk map. Relative risk is the ratio of the exposed group that will contract with the disease to unexposed group that will contract with the same disease. This paper discusses the most familiar approach in estimating the values of relative risk in the disease mapping; SMR model.

\section{MATERIALS AND METHOD}

\section{Standardized Morbidity Ratio (SMR)}

Based on [7], SMR method basically is the observed incidence divided by the expected incidence. The SMR method has been conventionally used for the counts within tracts analysis and it was calculated as follow:

$$
r_{i}=\hat{\theta}_{i}=\frac{o_{i}}{E_{i}}
$$

Here, $O_{i}$ is the number of observed incident cases or deaths of disease in the $i$ area while $E_{i}$ is referred as the number of expected cases.

Presume, for the study area to be mapped in disease mapping, it is partitioned into $M$ mutually exclusive states $(i=1,2, \ldots, M)$. Each states has its own number of observed cases $O_{i}$ and number of expected cases, $E_{i}$. In SMR model, to estimate the relative risk, $\hat{\theta}_{i}$ for area $i$, the values of $O_{i}$ and $E_{i}$ obtained from the existing data are used and defined as:

$$
r_{i}=\hat{\theta}_{i}=\frac{o_{i}}{E_{i}}
$$

We can find the number of observed cases from several sources for example health indicators from the website of Ministry of Health Malaysia. While, by using a formula as below, we can find the value of expected, $E_{i}$, for the study area:

$$
E_{i}=N_{i} \frac{\sum o_{j}}{\sum N_{j}}
$$

where $N_{i}$ is the population of region $i$ and the summations $(\Sigma)$ are for $j=1,2, \ldots, M$.

\section{Data Set}

The Department of Statistics and the Ministry of Health in Malaysia provided the data set used in this study. The SMR method is applied using this pneumonia data in the form of number of cases for 15 states in Malaysia; Perlis, Kedah, Penang, Perak, Federal Territory of Kuala Lumpur \& Putrajaya, Selangor, Negeri Sembilan, Melaka, Johor, Pahang, Terengganu, Kelantan, Sabah and Sarawak. The data received an annual time window. This paper used the observed new cases of infective in Malaysia, which is an aggregated data in year 2019. To compute the relative risk in this study, WinBUGS software is used and all these 
outcomes are presented in the form of graph and table. From the relative risk results, pneumonia risk map is constructed.

\section{RESULT AND DISCUSSION}

The relative risk estimation outcomes for all states in Malaysia are shown in Figure 2. From the graph, most of the states show a relative risk value below one for year 2019. In this analysis, the relative risk is referred as the conditional probability that a person in an area become infected with this disease divided by the conditional probability that a person in whole population become infected with this disease. According to Samat and Percy (2012), when a relative risk value close to 1 , it refers to no significant difference in terms of the likelihood of people suffering from pneumonia in an area and in the entire population. The people in an area generally less tending to be infected with this disease compared to people in the whole population when the value of relative risk is less than 1 . In contrast, if a relative risk value increase above 1 , this indicates that people in the area are more tending to be infected with this disease than people throughout the whole population. From Table 1, Negeri Sembilan and Terengganu have relative risk more than 1.50. This situation can conclude that people in these states have high tendency being infected with pneumonia than people in the whole population in Malaysia, while the other states are less prone to pneumonia than the entire Malaysia's population.

The comparison between the number of pneumonia cases recorded and the relative risk numerical values based on the SMR for year 2019 is shown in Table 1. From Table 1, the smallest observed cases are 529 people recorded for Labuan but the relative risk itself is not the smallest (1.1782) compared to the other relative risk estimates for pneumonia disease in 2019. Pulau Pinang has the lowest risk value of being infected with pneumonia with 0.5586 whereas Terengganu has the highest risk value of getting pneumonia with 1.6520. Thus, from some previous interpretations, it is not advisable to evaluate the spread of disease using only the observed cases. It is better to consider the relative risk estimation as it considers the transmission of disease in human populations.

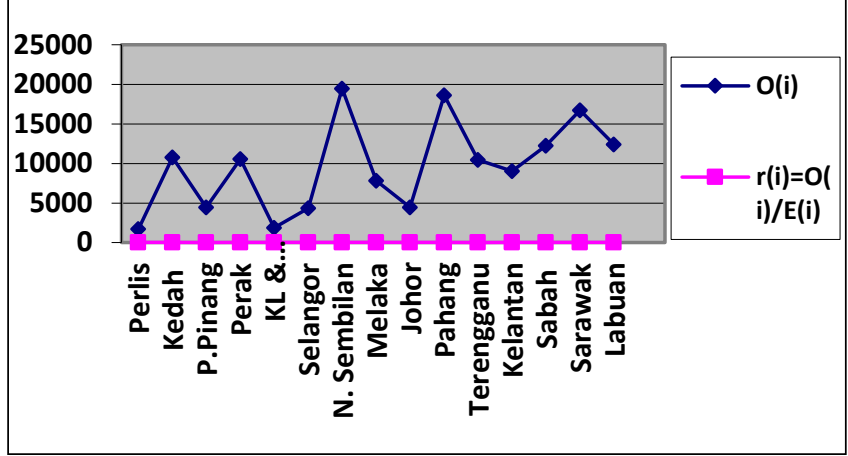

Figure 2. Plots of relative risk values estimated using the SMR and the number of cases for different states in Malaysia for year 2019

Table 1. Estimation of relative risk using SMR and frequency number of pnuemonia cases for year 2019

\begin{tabular}{|l|c|c|}
\hline States & Relative Risk & $\begin{array}{c}\text { Number of } \\
\text { Pneumonia Cases }\end{array}$ \\
\hline Perlis & 1.4605 & 1703 \\
Kedah & 1.0939 & 10760 \\
Penang & 0.5586 & 4447 \\
Perak & 0.9217 & 10550 \\
Kuala Lumpur \& Putrajaya & 0.7096 & 6208 \\
Selangor & 0.6820 & 19481 \\
Negeri Sembilan & 1.5219 & 7844 \\
Melaka & 1.0655 & 4442 \\
Johor & 1.0985 & 18617 \\
Pahang & 1.3747 & 10434 \\
Terengganu & 1.6520 & 9020 \\
Kelantan & 1.4908 & 12254 \\
Sabah & 0.9781 & 16728 \\
Sarawak & 0.9835 & 12402 \\
Labuan & 1.1782 & 529 \\
\hline
\end{tabular}

In this study, to show the distinction between high and low risk areas for the incidence of pneumonia cases in Malaysia, choropleth map is used. Each state is assigned one of five different levels of relative risk which are very low, low, medium, high and very high risks with respective interval of $[0.0,0.5),[0.5,1.0),[1.0,1.5),[1.5,2.0)$ and $[2.0, \infty)$. This interval division is chosen in order to analyse deeper as based on Samat and Percy (2012). The very low risk area is showed by the brightest colour of shade while very high risk area is indicated by the darkest colour. 


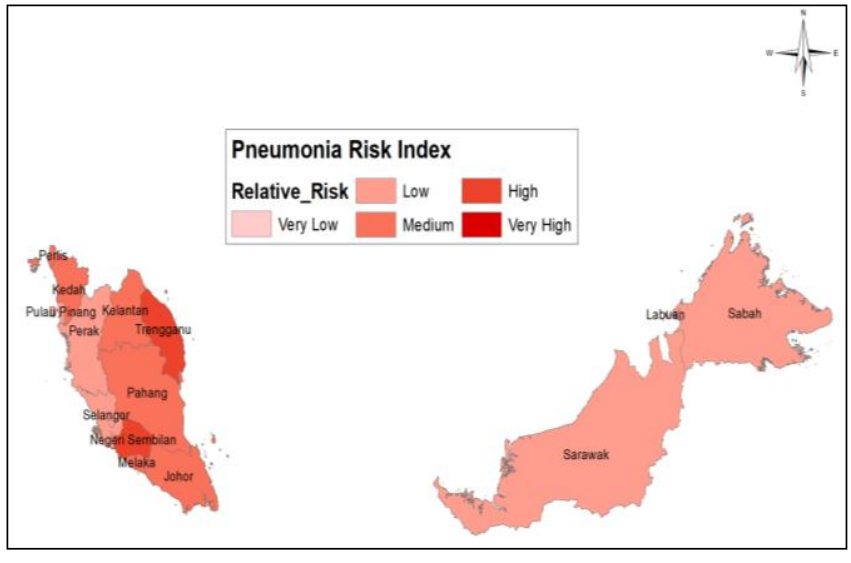

Figure 3. Map of relative risk values estimated using SMR method for year 2019

From the SMR map in Figure 3, it shows that the state of Negeri Sembilan and Terengganu are the high risk areas. Perlis, Kedah, Melaka, Johor, Pahang, Kelantan and Federal Territory of Labuan are categorized as medium risk areas of contracting pneumonia disease. While for other states other than those mentioned are low risk areas. There is no state classified as very low-risk and very high-risk area.

\section{CONCLUSION}

In monitoring and controlling pneumonia disease, estimation of the relative risk values is crucial. In this paper, researchers used SMR method to find the relative risk estimation which gives a better approach to evaluate the risk that accounted the population size (note: SMR method is not a forecasting approach for disease mapping). In conclusion, Terengganu has been recognized as the highest risk area with highest relative risk value which is 1.6520 while Pulau Pinang is the lowest risk area of being infected with pneumonia with relative risk value is 0.5586 . Though, direct use of SMR may not be practical as according to Lawson et al. (2003) and Meza (2003) as it has a few drawbacks. According to Meza (2003), SMR cannot detect the small areas as it does not take into consideration the high diversity of different regions and the spatial patterns of the areas under study. Since SMR is based on ratio estimators, the used of SMR can yield large changes in the estimate, and relatively small changes in expected value (Lawson et al., 2003). Therefore, this situation has inspired other researchers to offer new approaches in finding the relative risk estimation values.

\section{ACKNOWLEDGEMENT}

The authors would like to thank the Ministry of Health Malaysia and the Department of Statistics Malaysia for providing the data and Universiti Utara Malaysia for the support of this study.

\section{REFERENCES}

Department of Statistics Malaysia 2019, Statistics on Causes of Death, Malaysia, 2019, viewed 20 November 2020, <https://www.dosm.gov.my/v1/index.php?r=column/cth emeByCat\&cat=401\&bul_id=RUxlSDNkcnRVazJnakNCN VN2VGgrdzo9\&menu_id=LopheU43NWJwRWVSZklWdz Q4TlhUUTo9>.

Lawson, AB 2006, Statistical methods in spatial epidemiology, Wet Sussex, UK: John Wiley \& Sons, Ltd. Lawson, AB, Browne, WJ \& Rodeiro, CLV 2003, Disease mapping with WinBUGS and MLwiN, Statistics in Practise, Chichester: John Wiley \& Sons, Ltd.

Meza, JL 2003, 'Empirical Bayes estimation smoothing of relative risks in disease mapping', Journal of Statistical Planning and Inference, vol. 112, pp. 43-62.
Otieno, OJ, Joseph, M, \& John, O 2012, 'Mathematical model for pneumonia dynamics among children', The 2012 Southern Africa Mathematical Sciences Association Conference, (Samsa), pp. 26-29.

Rahil A 2019, Pneumonia: Symptoms, Causes, Treatment \& Vaccine, Bristol Medical Center, viewed 26 November 2020, <http://bristolmedicalcentre.com/pneumonia $>$.

Samat, NA \& Percy, DF 2012, 'Dengue disease mapping in Malaysia based on stochastic SIR models in human populations', in ICSSBE 2012: Proceedings of 2012 International Conference on Statistics in Science, Business and Engineering: "Empowering Decision Making with Statistical Sciences," pp. 623-627.

World Health Organization (WHO) 2019, Pneumonia Fact Sheet, viewed 25 November 2020, 
$<$ http://www.who.int/news-room/factsheets/detail/pneumonia>.

World Health Organization 2020, Global research on coronavirus disease (COVID-19), viewed 25 November 2020,

<https://www.who.int/emergencies/diseases/novel-

coronavirus-2019/global-research-on-novel-coronavirus2019-ncov>. 